\title{
Encoding specificity: The case of maps and text
}

\author{
RAYMOND W. KULHAVY and WILLIAM A. STOCK \\ Arizona State University, Tempe, Arizona \\ SARAH E. PETERSON \\ Northern Illinois University, De Kalb, Illinois \\ and \\ REBECCA BROOKS \\ Armstrong Laboratories, Williams Air Force Base, Arizona
}

\begin{abstract}
Undergraduates studied either an intact reference map or a random distribution of map features and then listened to a text that contained facts related to the map. Next, half of the subjects in each encoding group received the map or the distribution of features and used these materials as retrieval cues for recalling the text facts. Seeing the intact map prior to hearing the text led to better fact recall, and there was no effect for the type of cue presented at retrieval. The results provide no support for encoding specificity in the case in which a spatial display such as a map is used to improve memory for text.
\end{abstract}

We investigated how people use spatial displays to improve memory for verbal materials associated with the displays. For example, successful retrieval of text-based facts is considerably higher when subjects study a related map than when they see a list of map features or no map at all (see, e.g., Schwartz \& Kulhavy, 1981). These results suggest that it is the intact property of the map display that is important for retrieval of associated facts.

How does the study of an intact map improve recall of text facts? We have adopted a position based in dualcoding theory (Paivio, 1971, 1986), in which maps are encoded as images that retain information present in the stimulus map itself and text information is represented in a nonimage verbal code. There are access links between the two codes, and information from the map image can be used as cross-code retrieval cues for facts contained in the verbal store. Use of information from the map image to cue retrieval of associated verbal content explains why map learning facilitates text retrieval. We have developed the details of this position in a recent paper (Kulhavy, Stock, Woodard, \& Haygood, in press).

Maps encoded as images allow people to access two types of information. First, there is feature information, which consists of attributes of the individual features on the map, including properties such as shape, size, and color (Bertin, 1983). Feature information has been the focus of most work on memory for pictures and prose (Willows \& Houghton, 1987). Second, map images contain structural information, which represents the coordinate and metric relationships among features in the map

Correspondence should be addressed to R. W. Kulhavy, Applied Cognition Facility, Payne Hall-0611, Arizona State University, Tempe, AZ 85287-0611. space. When map features are encoded as individual entities in memory, they can be used to cue related verbal units. However, it requires considerable working-memory space to retrieve each unit and use it effectively. On the other hand, when a map is encoded as an intact unit, structural information is added to the image and the entire display can be processed simultaneously without exceeding the resources of the active-memory system. The computational advantage of being able to shift attention from location to location across intact map images explains why intact stimulus maps improve text recall more than does presentation of individual features (see, e.g., Larkin \& Simon, 1987; Paivio, 1986). The probability of accessing appropriate cross-code cuing information is higher when the map image is represented as an intact unit.

We have recently completed an experiment in which people studied maps, heard a related text, and then received as a retrieval cue either the original intact map or a version with feature locations changed (Kulhavy, Stock, Peterson, Pridemore, \& Klein, 1992, Experiment 1). Generally, subjects who received the intact version of the original map remembered more text events than did subjects seeing the map with feature locations changed. We attributed this effect to the fact that the new feature order interfered with the encoded map image at retrieval. In other words, the rearranged map did not contain the structural information originally encoded, and subjects viewing that map were more likely to use the individual features as cues for text-fact retrieval.

Several of our colleagues (including journal reviewers) have pointed out to us that the results described above are more parsimoniously explained by the principle of encoding specificity (Tulving \& Thomson, 1973). In this case, the intact retrieval map provided the same stimulus context present at encoding and, therefore, led to better 
recall of the text. We were unable to find evidence for encoding specificity in a second experiment reported in Kulhavy et al. (1992), in which we varied the form of map-based retrieval cues. We believe that a clearer test is required to differentiate encoding specificity from the results predicted by the dual-coding model. Hence, we designed the current experiment, which pitted intact maps against a random distribution of features during both encoding and retrieval and measured the effects of these combinations on the recall of facts from a related text.

Encoding specificity predicts higher recall of text facts when the spatial stimuli are the same at both encoding and retrieval. Consequently, both the map-map and feature-feature conditions should produce higher fact retrieval than either of the two conditions in which the spatial stimulus changes from encoding to retrieval (i.e., map-feature and feature-map). This expectation follows from the central principle that the stimulus context present at encoding will be the most successful vehicle for retrieval (Tulving, 1983).

The predictions stemming from our dual-coding approach are somewhat different. Because of the computational advantage that occurs when maps are encoded as intact units, we agree that the map-map condition will yield the best text-retrieval performance. However, the same prediction does not apply to the case in which subjects initially study a distribution of features and see them again at retrieval. We believe that encoding maps as intact images is an act of semantic memory, heavily influenced by prior experience with maps themselves. When people are faced with a page of map features, our guess is that they are less likely to encode them as an entity and more likely to try to learn them as discrete units. The result, of course, is a less efficient encoding, with a consequent reduction in the number of map-image cues available for cross-code retrieval. Consequently, we expect the feature-feature condition will produce retrieval of fewer facts than will the map-map condition. Finally, encoding specificity and dual coding make essentially the same prediction for the remaining two groups, in which the type of stimulus display changes from encoding to retrieval. One possible exception for dual coding is that the map-feature group may produce higher text recall than the feature-map group, simply because the map was encoded as an intact unit in the first place and may retain some of the advantage due to structural information. This advantage may persevere in spite of the fact that these subjects see the distribution of features at encoding.

\section{METHOD}

\section{Design and Subjects}

The experiment crossed two levels of learning and retrieval cues with both immediate and delay testings. The base design was a 2 learning cue (organized map vs. distributed features) $\times 2$ retrieval cue (organized map vs. distributed features) $\times 2$ color (modified by color word vs. not modified) $\times 2$ test occasion (immediate vs. one-week delay) factorial, with repeated measures on the color and test-occasion variables.

The subjects were 87 undergraduate volunteers attending a large university in the midwestern United States. The subjects were randomly assigned to conditions on the basis of the order in which they appeared for the experiment. Three subjects failed to complete all experimental tasks successfully, and their data were dropped from the analyses. There were 23 subjects in the group that learned the organized map and were cued with distributed features and 21 subjects in the remaining three conditions.

\section{Materials}

The organized map represented a Roman town of the classic period. The map contained 17 landmark features distributed within a wall surrounding the city, with an unnamed road bisecting the city from west to east. Each of the 17 features was depicted by a line drawing and included a label printed directly below it. The 17 feature labels were Appia Street, Arch, Barracks, Bath House, Cafe, Capitol, Cemetery, Fountain, Garden, Gate, Market, Monument, Plaza, Statue, Temple, Via Rosa, and Watch Tower. For the distributed-features condition, the wall and the west-east road were removed and the 17 feature landmarks were randomly distributed within the space originally surrounded by the wall. For use in the study, both the organized map and the page of features were printed in black ink on a sheet of $21.6 \times 27.9 \mathrm{~cm}$ white paper.

The fact text contained about 800 words and consisted of 38 sentences, 34 of which contained fact information and 4 of which were filler sentences ( 2 at the beginning and 2 at the end) used to make the text more comprehensible. Each of the landmark features on the map was mentioned in 2 sentences in the text, with a different fact linked to the feature in each sentence. There were no obvious associations between the 34 facts. Of the 2 sentences for a given map feature, 1 contained a color word modifying the feature name and the other presented the feature name without a color word. No color was used for more than 1 feature. Examples of both the color-modified and nonmodified sentences for the feature cafe are presented below.

The blue cafe is the location where the rare public executions take place in the city. (modified)

Over at the cafe the scribes of the town maintain a written history of the city. (nonmodified)

The 34 feature-fact sentences were assigned in two random orders to positions in the passage, with the restrictions that no 2 occurrences of a specific feature were adjacent to one another and that color-modified and nonmodified sentences appeared in alternate order throughout the text. Which of the two orders was heard by the subjects was randomly determined prior to the experimental session. The passage was recorded in a female voice at approximately 100 words per minute for use in the study.

\section{Procedure}

The subjects participated in groups, with the subjects from each between-subject condition about equally represented in every session. As they entered the room, the subjects were given an envelope from a randomly shuffled stack that assigned them to their particular condition. The subjects were seated as far as possible from one another to preclude their seeing another person's materials. Once all the subjects were seated, an experimenter read general instructions describing the experimental sequence and then answered procedural questions. Next, each subject removed the first page, which contained either the organized map or the page of features, from his/her envelope and was instructed to study the page carefully. The subjects were told that learning the first page of material would assist them in learning information to be presented later in the study. The subjects studied the map or page of features for $2 \mathrm{~min}$.

Following the 2 min of study, the subjects were told to use the map to help them learn the text information they would hear next. The subjects kept their map or page of features in front of them as they listened to two playings of the tape. Instructions to use the information on the page to help them learn the text were repeated between playings. Following the tape, all materials were collected and the subjects spent 3 min working on a page of arithmetic problems to reduce direct recall from active memory. Following the interpolated task, the subjects were given either the organized map or the page of features (depending on their condition) and three sheets of blank white paper. The subjects were told to write down everything they could remember from the text played 
on the tape. This free recall was untimed. Exactly 1 week later, the subjects returned to the room, were given the map or features, and were given unlimited time to complete a second recall of the text.

\section{RESULTS AND DISCUSSION}

A text fact was scored as correct and awarded 1 point if the substance of the fact was included in the response. The written fact protocols were independently scored by 2 people, with an agreement of $87 \%$ between the 2 scorers. In the results reported below, all statistical tests were evaluated at $p<.05$.

Proportion correct fact recall was analyzed using a 2 (learning cue) $\times 2$ (retrieval cue) $\times 2$ (color) $\times 2$ (occasion) analysis of variance. There were significant main effects for both color $\left[F(1,142)=76.41, M S_{\mathrm{e}}=.02\right]$ and occasion $\left[F(1,142)=5.96, M S_{\mathrm{e}}=.01\right]$ and for the color $\times$ occasion interaction $\left[F(1,142)=4.48, M S_{\mathrm{e}}=.01\right]$. These effects were subsumed by a significant interaction of learning cue $\times$ color $\times$ occasion $[F(1,142)=3.89$, $\left.M S_{\mathrm{e}}=.01\right]$. No other main effect or interaction reached significance. The means and standard deviations contributing to this analysis are presented in Table 1.

In general, facts not modified by a color word were remembered better, and, of course, immediate test performance was superior to delay. The significant three-way interaction is due to the fact that the group that saw the

Table 1

Means and Standard Deviations for Proportion of Text Facts Recalled

\begin{tabular}{|c|c|c|c|c|c|}
\hline \multirow[b]{4}{*}{ Condition } & & \multicolumn{4}{|c|}{ Encoding Stimulus } \\
\hline & & \multirow{2}{*}{\multicolumn{2}{|c|}{$\begin{array}{c}\text { Feature } \\
\text { Retrieval }\end{array}$}} & \multirow{2}{*}{\multicolumn{2}{|c|}{$\begin{array}{c}\text { Map } \\
\text { Retrieval }\end{array}$}} \\
\hline & & & & & \\
\hline & & Feature & Map & Feature & Map \\
\hline \multicolumn{6}{|c|}{ Immediate Test } \\
\hline \multirow[t]{2}{*}{ Color } & $M$ & .54 & .56 & .53 & .58 \\
\hline & $S D$ & .22 & .22 & .23 & .23 \\
\hline \multirow[t]{2}{*}{ No Color } & $M$ & .61 & .62 & .64 & .70 \\
\hline & $S D$ & .24 & .23 & .20 & .20 \\
\hline \multicolumn{6}{|c|}{ Delayed Test } \\
\hline \multirow[t]{2}{*}{ Color } & $M$ & .40 & .44 & .46 & .48 \\
\hline & $S D$ & .24 & .22 & .21 & .23 \\
\hline \multirow[t]{2}{*}{ No Color } & $M$ & .47 & .51 & .52 & .57 \\
\hline & $S D$ & .25 & .23 & .21 & .23 \\
\hline
\end{tabular}

intact map at encoding remembered a greater number of color-modified facts on the delay test.

Although these results are not those predicted in our initial discussion, they do provide some interesting information. There is no support for the principle of encoding specificity. For text recall, it made no difference whether the retrieval cue was the same as or different from that seen during encoding. In fact, the type of retrieval cue failed to contribute to any of the effects examined in the analysis. The dominant factor was the spatial stimulus seen during encoding. Generally, fact recall was higher when the subjects encoded the intact map, and these subjects remembered proportionately more facts after a 1-week delay. These data appear to fit a dual-coding explanation better than one based in encoding specificity.

This experiment provides no firm conclusions regarding how map images interact with verbal information during retrieval. However, the recall advantage for intact maps suggests that encoding specificity may not extend directly to the case in which maplike displays are represented and processed by the cognitive system.

\section{REFERENCES}

BERTIN, J. (1983). Semiology of graphics: Diagrams, networks, maps (W. J. Berg, Trans.). Madison: University of Wisconsin Press.

Kulhavy, R. W., Stock, W. A., Peterson, S. E., Pridemore, D. R., \& KLeIN, J. D. (1992). Using maps to retrieve text: A test of conjoint retention. Contemporary Educational Psychology, 17, 56-70.

Kulhavy, R. W., Stock, W. A., Woodard, K. E., Haygood, R. C. (in press). Comparing elaboration and dual coding theories: The case of maps and text. American Journal of Psychology.

LARKIN, J. H., Simon, H. A. (1987). Why a diagram is (sometimes) worth ten thousand words. Cognitive Science, 11, 65-99.

Paivio, A. (1971). Imagery and verbal processes. New York: Holt, Rinehart \& Winston.

Paivio, A. (1986). Mental representations. New York: Oxford University Press

SchwartZ, N. H., Kulhavy, R. W. (1981). Map features and the recall of discourse. Contemporary Educational Psychology, 6, 151-158.

Tulving, E. (1983). Elements of episodic memory. New York: Oxford University Press.

Tulving, E., \& Thomson, D. M. (1973). Encoding specificity and retrieval processes in episodic memory. Psychological Review, 80, 352-373.

Willows, D. M., \& Houghton, H. A. (1987). The psychology of illustration (Vol. 1). New York: Springer-Verlag.

(Manuscript received October 9, 1992.) 